Nonlinear Processes in Geophysics, 12, 835-848, 2005

SRef-ID: $1607-7946 / \mathrm{npg} / 2005-12-835$

European Geosciences Union

(C) 2005 Author(s). This work is licensed

under a Creative Commons License.

\title{
Extracting preseismic electromagnetic signatures in terms of symbolic dynamics
}

\author{
K. Karamanos ${ }^{1}$, A. Peratzakis ${ }^{2}$, P. Kapiris ${ }^{2}$, S. Nikolopoulos ${ }^{3}$, J. Kopanas ${ }^{2}$, and K. Eftaxias ${ }^{2}$ \\ ${ }^{1}$ Center for Nonlinear Phenomena and Complex Systems, Universite Libre de Bruxelles, CP231 Campus Plaine, 1050, \\ Bruxelles, Belgium \\ ${ }^{2}$ Department of Physics, Section of Solid State Physics, Univ. of Athens, Panepistimiopolis, 15784 Zografos, Athens, Greece \\ ${ }^{3}$ National Technical Univ. Athens, Dept. of Electrical and Computer Engineering Zografou Campus, 15773 Zografou, Greece
}

Received: 2 May 2005 - Revised: 3 August 2005 - Accepted: 3 August 2005 - Published: 20 September 2005

\begin{abstract}
When a heterogeneous material is strained, its evolution toward breaking is characterized by the nucleation and the coalescence of micro-cracks before the final breakup. Electromagnetic (EM) emission in a wide frequency spectrum ranging from very low frequencies (VLF) to very high frequencies (VHF) is produced by micro-cracks, which can be considered as the so-called precursors of general fracture. Herein we consider earthquakes (EQs) as large-scale fracture phenomena. We study the capability of nonlinear time series analysis to extract features from pre-seismic electromagnetic (EM) activity possibly indicating the nucleation of the impending EQ. In particular, we want to quantify and to visualize temporal changes of the complexity into consecutive time-windows of the time series. In this direction the original continuous time EM data is projected to a linguistic symbolic sequence and then we calculate the block entropies of the optimal partition. This analysis reveals a significant reduction of complexity of the underlying fractoelectromagnetic mechanism as the catastrophic events is approaching. We verify this result in terms of correlation dimension analysis. We point out that these findings are compatible with results from an independent linear method which uses a wavelet based approach for the estimation of fractal spectral characteristics. Field and laboratory experiments associate the epoch of low complexity in the tail of the precursory emission with the nucleation phase of the impending earthquake.
\end{abstract}

\section{Introduction}

One of the basic problems of human existence is the prediction of future events. A vital problem in material science and

Correspondence to: K. Eftaxias

(ceftax@phys.uoa.gr) in geophysics is the identification of precursors of macroscopic defects or shocks. In physics, the degree to which we can predict a phenomenon is often measured by how well we understand it. Despite the large amount of experimental data and the considerable effort that has been undertaken by the material scientists, many questions about the fracture remain standing. Herein, by combining nonlinear and linear methods of analysis, we hope to uncover more information hidden in the pre-seismic EM time series, and thus, to achieve a deeper understanding of the physics of the EQ nucleation process.

When a heterogeneous material is strained, its evolution toward breaking is characterized by the nucleation and the coalescence of micro-cracks before the final break-up. EM emission in a wide frequency spectrum ranging from very low frequencies (VLF) to very high frequencies (VHF) is produced by opening micro-cracks, which can be considered as the so-called precursors of general fracture. These precursors are detectable both at a laboratory and a geological scale. It is by now recognized that the pre-fracture VLF-VHF EM time-series contain valuable information about the fracture/EQ preparation process.

In this paper, we are studying in terms of nonlinear and linear techniques, whether precursory signatures emerged indicating the transition to the last phase of the EQ preparation process. More precisely, first the temporal evolution of nonlinear characteristics is studied by applying a recently proposed technique: the original continuous time EM data is projected to a symbolic sequence and a block entropy analysis by lumping follows. This analysis suggests as main result that as the last phase of EQ preparation process (nucleation phase) evolves there is a clear transition from higher to lower complexity. We verify this result in terms of a different nonlinear technique, namely, "correlation dimension analysis". As it will be shown in the sequel, the results of the 
afore-mentioned two nonlinear methods are compatible. It would be highly desirable to confirm the above-mentioned conclusion based on an independent linear fractal spectral analysis. By monitoring the temporal evolution of the fractal spectral characteristics on pre-seismic EM time series we show that significant alterations in associated scaling parameters emerge as global failure is approaching. These alterations also reveal that the significant reduction of complexity, in the tail of the pre-seismic EM activity, is accompanied by the transition from anti-persistent to persistent behavior. This convergence between nonlinear and linear analysis provides a more reliable detection concerning the emergence of the last (nucleation) phase of the EQ preparation process. Direct laboratory and field experimental data support the conclusions of the present analysis. The identification of such a transition state is of special interest for the understanding of mechanisms generating EQs.

The paper is organized as follows. In Sect. 2 we refer to some prerequisites. Firstly, we present some generalities concerning EM emissions prior to fracture from the laboratory to the geophysical scale and continue with data collection information. In the next section, we have to recall some basic facts about symbolic sequences and block entropy. Section 4 will be devoted to the application of the afore-mentioned concept to pre-seismic EM recordings. The general formulation of the Correlation Dimension $D_{2}$ analysis is laid down in Sect. 5, as well as, its application to the data under study. In Sect. 6 the linear method of fractal spectral analysis is presented and applied for the case of the Athens EQ. Finally in the last section we draw the main conclusions and discuss potential improvements of the analysis.

\section{Prerequisites}

\subsection{EM emissions from fracture}

Crack propagation is the basic mechanism of material's failure. The motion of a crack in dynamics fracture has been shown to be governed by a dynamical instability causing oscillations in its velocity and structure on the fracture surface. Experimental evidence indicates that the instability mechanism is that of local branching (Sharon et al., 1995; Sharon and Fineberg, 1996): a multi-crack state is formed by repetitive, frustrated micro-fracturing events (Sharon and Fineberg, 1999).

In many materials, emission of photons, electrons, ions and neutral particles are observed during the formation of new surface features in fracturing, deformation, wearing, peeling, and so on. Collectively, we refer to these emissions as fracto-emission (Langford et al., 1987; Dickinson et al., 1988; Gonzales and Pantano, 1990; Miura and Nakayama, 2000; Bahat et al., 2002). It is worth mentioning that laboratory experiments show that more intense fracto-emissions are observed during the unstable crack growth (Gonzales and Pantano, 1990). The rupture of inter-atomic (ionic) bonds also leads to intense charge separation that is the origin of the electric charge between the micro-crack faces. On the faces of a newly created micro-crack the electric charges constitute an electric dipole or a more complicated system. Due to the crack strong wall vibration in the stage of the microbranching instability, it behaves as an efficient EM emitter. These EM precursors are detectable both at a laboratory (Frid et al., 1999; Rabinovitch et al., 2002, Mavromatou et al., 2004; Eftaxias et al., 2004 and references therein) and geological scale (Hayakawa, 1999; Hayakawa and Molchanov, 2002; Gershenzon and Bambakidis, 2001; Contoyiannis et al., 2005). Our main tool is the monitoring of the microfractures, which possibly occur in the pre-focal area before the final break-up, by recording their EM emissions. A multidisciplinary analysis in terms of fault modelling (Eftaxias et al., 2001), laboratory experiments (Eftaxias et al., 2002), scaling similarities of multiple fracturing of solid materials (Kapiris et al., 2004a), fractal electrodynamics (Eftaxias et al., 2004), criticality (Kapiris et al., 2004b, Contoyiannis et al., 2005), and complexity (Kapiris et al., 2005a), seems to validate the association of the detected pre-seismic EM emissions with the fracturing process in the pre-focal area of the impending EQ.

\subsection{Data collection}

Aiming at recording electromagnetic precursors, since 1994 a station was installed at a mountainous site of Zante island $\left(37.76^{\circ} \mathrm{N}-20.76^{\circ} \mathrm{E}\right)$ in western Greece. An important EQ $\left(M_{s}=5.9\right)$ occurred on 7 September 1999 at 11:56 GMT at a distance of about $20 \mathrm{~km}$ from the center of the city of Athens, the capital of Greece. Very clear EM anomalies have been detected in the VLF band, i.e. by the six loop antennas detecting the three components (East-West, North-South, and vertical) of the variation of the magnetic field at $3 \mathrm{kHz}$ and $10 \mathrm{kHz}$, before the Athens EQ (Eftaxias et al., 2001; Kapiris et al., 2005b), before the Athens EQ. In Fig. 1 we present the EM time series at $10 \mathrm{kHz}$ from 4 July 1999 up to 12 September 1999. In Fig. 2, we show the same EM time series on 28 August 1999 and from 4-7 September 1999. The whole EM precursors were emerged a few days prior to the event. They are characterized by an accelerated emission rate, while, this radiation is embedded in a long duration quiescence period. These emissions have a rather unanticipated long duration, (a few days) while the sampling rate was $1 \mathrm{sample} / \mathrm{sec}$, and thus they provide sufficient data for statistical analysis. The seismogenic origin of this EM activity has been supported by a series of previous papers (Eftaxias et al., 2001, 2004; Kapiris et al., 2004a, 2005a, b; Contoyiannis et al., 2005).

\section{Dynamical characteristics of pre-seismic EM activity in terms of block entropies by lumping}

A way to examine transient phenomena is to analyze the preseismic EM time series into a sequence of distinct time windows. The aim is to discover a clear difference of dynamical 


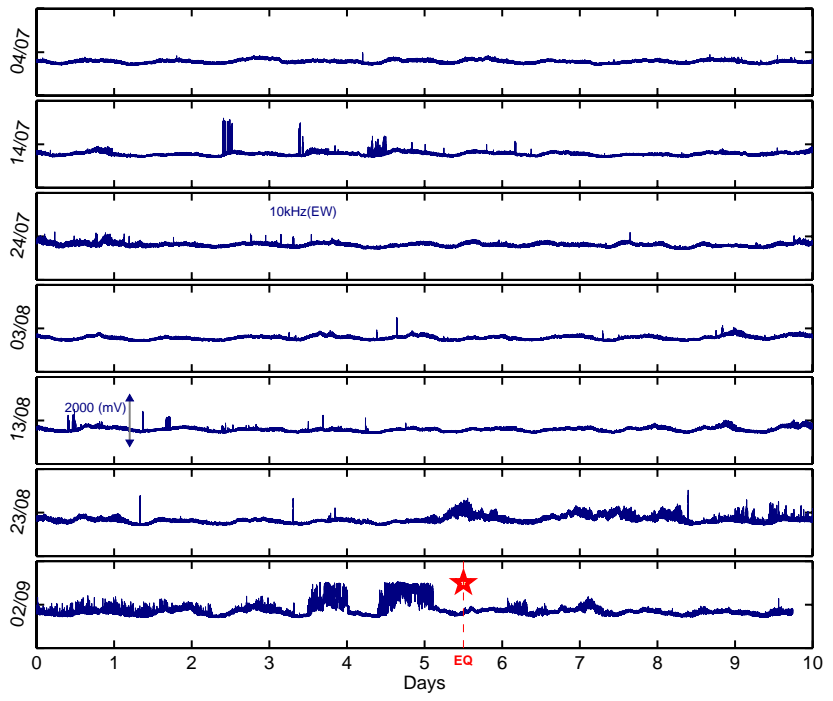

Fig. 1. Time-series of the $10 \mathrm{kHz}$ (East-West) magnetic field strength between 4 July 1999 and 11 September 1999 in arbitrary units. The star indicates the time of the Athens earthquake occurrence.

characteristic as the catastrophic event is approaching. In this section, we apply ideas from symbolic dynamics. In particular, block entropies by lumping analysis is applied. Within each time window, the block entropy serves as a measure of "complexity" of the signal: the lower the value of entropy, the more "ordered" it is. Our results suggest that an important principle, i.e. substantial complexity decrease prior to a significant $\mathrm{EQ}$, can be confirmed. In the following we will shortly review the concepts of symbolic dynamics and block entropy.

We note that Schwarz et al. (1993) have used the methods of Symbolic Dynamics in a very similar way in the analysis of solar spike events.

\subsection{Symbolic dynamics}

The discovery that simple deterministic systems can show a vast richness of behaviors in response to variations of initial conditions and /or control parameters, has been at the origin of an intense interdisciplinary research activity during the last two decades (Khinchin, 1957; Nicolis, 1991, 1995). One of the outcomes of this work has been the realization that for an appropriate description of such complex systems, one needs to resort to a probabilistic approach (Nicolis and Gaspard, 1994). Now, once one leaves the description in terms of trajectories, a basic question that must be dealt with concerns the amount of information one may have access to on the evolution of the system in the course of time. One of the approaches developed in this context is coarse-graining, whereby a complex system is viewed as an information generator producing messages constituted of a discrete set of symbols defined by partitioning the full continuous phase space into a finite number of cells. We refer to such a description as "symbolic dynamics" (Nicolis et al., 1989; Nico-
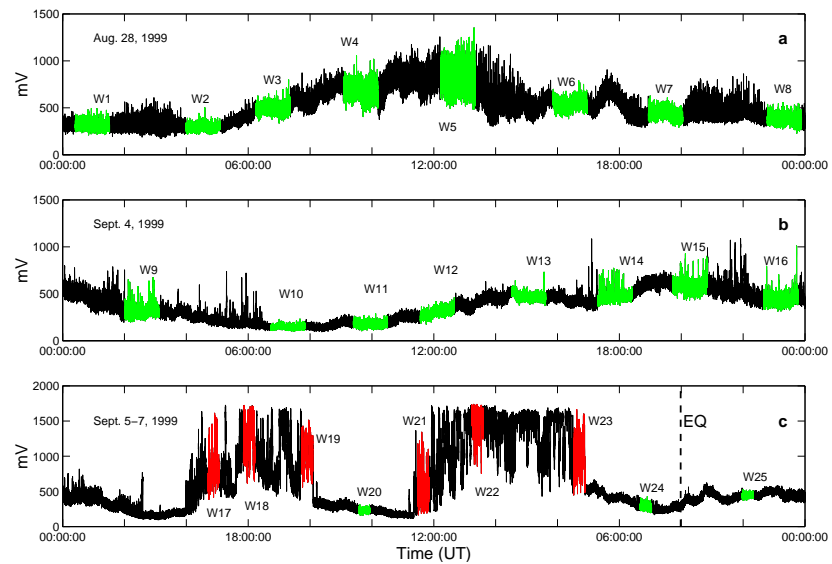

Fig. 2. View of segments of the time-series of the $10 \mathrm{kHz}$ (EastWest) magnetic field strength depicted in Fig. 1. The vertical line indicates the time of the Athens earthquake occurrence. We note the emergence of two strong impulsive signals in the tail of the precursory emission. These anomalies are embedded in a long duration quiescence period concerning the detection of EM disturbances at the VLF frequency band.

lis, 1991, 1995; Nicolis and Gaspard 1994). One of its merits is to provide a link between dynamical systems, information theory, and cognitive sciences (Nicolis, 1991; Ebeling and Nicolis, 1992).

There exist some canonical ways for generating symbolic dynamics out of a given dynamical system (Nicolis, 1991, 1995, 1988, 1989; Ebeling and Nicolis, 1992). Our objective in the present work is to analyze preseismic EM emissions. Block entropies are subsequently calculated, clearly reflecting the subtle interlay of the transfer of information between the "syntactic" and the "grammatical" levels (Nicolis, 1991).

To produce symbolic dynamics out of the evolution of a given system, we set up a coarse-grained description incorporating from the very beginning the idea that a physically accessible state corresponds to a finite region rather than to a single point of phase space. Let $C_{i}(i=1,2, \ldots K)$ be the set of cells in phase space constituted by these regions, assumed to be connected and non-overlapping. As time goes on, the phase space trajectory performs transitions between cells thereby generating sequences of $K$-symbols, which may be regarded as the letters of an alphabet. We shall require that, in the course of these transitions, each element of the partition is mapped by the law of evolution on a union of elements.

We restrict ourselves to the simplest possible coarse graining of the preseismic signal. This is given by choosing a threshold $C$ and assigning the symbols " 1 " and " 0 " to the signal, depending on whether it is above or below the threshold (binary partition). In this way, each stationary time window of the original EM time-series for a given threshold is transformed into symbolic sequences, which contains "linguistic" or "symbolic dynamics" characteristics. 


\subsection{The dynamical (Shannon-like) block entropy}

Block entropies, depending on the word-frequency distribution, are of special interest, extending Shannon's classical definition of the entropy of a single state to the entropy of a succession of states (Nicolis and Gaspard, 1994; Karamanos and Nicolis, 1999). Each entropy takes a large (small) value if there are many (few) kinds of patterns, therefore, it decreases while the organization of patterns is increasing. In this way, the block entropy can measure the complexity of a stationary signal.

In particular, we estimate the block entropies by lumping. Lumping is the reading of the symbolic sequence by "taking portions", as opposed to gliding where one has essentially a "moving frame". In general, the basic novelty of the entropy analysis by lumping is that, unlike the Fourier transform or the conventional entropy by gliding, it gives results that can be related to algorithmic aspects of the sequences.

\subsection{Entropy analysis by lumping}

It is useful to transform the initial raw data of the EM signal into symbolic sequences taking values in the alphabet $\{0,1\}$, according to the rules $A_{i}=1$ if $A\left(t_{i}\right)>E\left[A\left(t_{i}\right)\right]$ and $A_{i}=0$, if $A\left(t_{i}\right)<E\left[A\left(t_{i}\right)\right]$, where $A\left(t_{i}\right)$ are the values of the measured field at time $t_{i}$ and $E\left[A\left(t_{i}\right)\right]=<A\left(t_{i}\right)>$ is the mean value in the particular stationary time windows, as it is nicely stated in Schwarz et al. (1993).

Consider a subsequence of length $N$ selected out of a very long (theoretically infinite) symbolic sequence. We stipulate that this subsequence is to be read in terms of distinct "blocks" of length $n$,

$\cdots \underbrace{A_{1} \ldots A_{n}}_{B_{1}} \underbrace{A_{n+1} \ldots A_{2 n}}_{B_{2}} \ldots \underbrace{A_{j n+1} \ldots A_{(j+1) n}}_{B_{j+1}} \cdots$

We call this reading procedure "lumping". We shall follow lumping in the sequel.

The following quantities characterize the information content of the sequence (Khinchin, 1957; Ebeling and Nicolis, 1992):

1. The dynamical (Shannon-like) block-entropy for blocks of length $n$

$$
H(n)=-\sum_{\left(A_{1}, \ldots, A_{n}\right)} p^{(n)}\left(A_{1}, \ldots, A_{n}\right) \cdot \ln p^{(n)}\left(A_{1}, \ldots, A_{n}\right)
$$

where the probability of occurrence of a block $A_{1} \ldots A_{n}$, denoted $p^{(n)}\left(A_{1}, \ldots, A_{n}\right)$, is defined by the fraction (when it exists) in the statistical limit as

$$
\frac{\text { No. of blocks, } A_{1} \ldots A_{n} \text {, encountered when lumping }}{\text { total No. of blocks }}
$$

starting from the beginning of the sequence, and the associate entropy per letter.

$$
h^{(n)}=\frac{H(n)}{n} .
$$

2. The conditional entropy or entropy excess associated with the addition of a symbol to the right of an $n$-block

$$
h_{(n)}=H(n+1)-H(n) .
$$

3. The entropy of the source (a topological invariant), defined as the limit (if it exists)

$$
h=\lim _{n \rightarrow \infty} h_{(n)}=\lim _{n \rightarrow \infty} h^{(n)}
$$

which is the discrete analog of metric or Kolmogorov entropy.

We now turn to the selection problem that is to the possibility of emergence of some preferred configurations (blocks) out of the complete set of different possibilities. The number of all possible symbolic sequences of length $n$ (complexions in the sense of Boltzmann) in a $K$-letter alphabet is

$N_{K}=K^{n}$.

Yet not all of these configurations are necessarily realized by the dynamics, nor are they equiprobable. A remarkable theorem due to McMillan (Khinchin, 1957; Nicolis and Gaspard, 1994), gives a partial answer to the selection problem asserting that for stationary and ergodic sources the probability of occurrence of a block $\left(A_{1}, \ldots, A_{n}\right)$ is

$p_{n}\left(A_{1}, \ldots, A_{n}\right) \sim e^{-H(n)}$

for almost all blocks $\left(A_{1}, \ldots, A_{n}\right)$. In order to determine the abundance of long blocks one is thus led to examine the scaling properties of $H(n)$ as a function of $n$.

As we have already mentioned, the Fourier spectrum or the standard convention of the entropy analysis by gliding, do not help us to distinguish between symbolic sequences with completely different levels of complexity and spectra (Karamanos, 2001). Unlike the previous methods, the novelty of the entropy analysis by lumping gives results, which can be connected with algorithmic aspects of the sequences, in particular with the property of the sequence to be generated by deterministic or stochastic automata (see Karamanos, 2001). Also, the entropy analysis by lumping of some weakly chaotic systems, gives a rather characteristic entropy spectrum, as explained in (Karamanos, 2001). This shows that the entropy analysis by lumping is much more sensitive in algorithmic and ergodic properties of (weakly) chaotic systems than the classical conventional entropy analysis by gliding, or the correlation functions.

\section{Results in terms of symbolic dynamics}

\subsection{Stationarity of the mean value}

We recall that the block entropy can measure the complexity of a stationary signal. Thus, starting from the raw data, we first isolate stationary windows, if they exist, in different distinct epochs of the pre-seismic EM-series. 


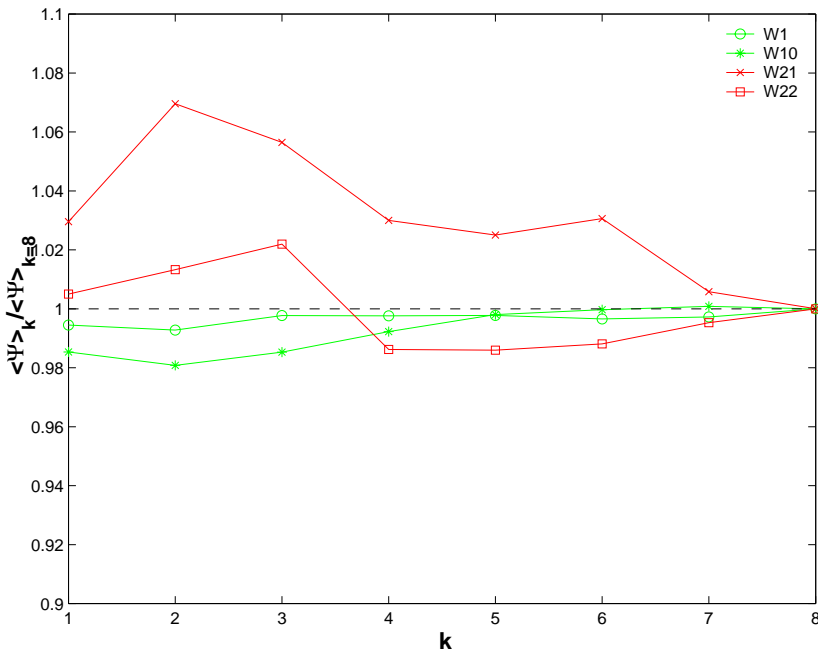

Fig. 3. The stationarity test for the typical windows W1, W10, W21 and W22. We divide each time window into sub-regions, which have common center the middle of each time window and a radius $R=k \cdot 250$ points, with $\mathrm{k}=1$ to 8 . In the vertical axis, we present the ratio of the mean value of the sub-window divided by the mean value of the whole window. The stationarity is clear. (For long enough sub-windows the deviation from the mean value of the main window is smaller than $5 \%$.)

First, we search for locally stationary and long enough time windows of the pre-seismic EM time series as the main event is approaching. As the test of stationarity we have run the following algorithm. Starting from the middle of the window, we consider some sub-windows around the center with an increasing radius. We consider that a window is stationary when the variation of the mean values of the sub-windows is not very important(less than 5\%). In Fig. 2 we isolate 25 windows which present a very good stationary behavior according to the previous test. To be concrete, in Fig. 3 we characteristically depict the aforementioned check for three time windows.

We emphasize that large amounts of data ensure the convergence to the statistical limit, and so, higher precision of the results. Especially in non-linear methods, the amount of data is a crucial issue. In our study the length of the observation windows is of 4000 samples. This ensures a good statistical precision.

\subsection{Optimal partition}

It is well known that the real block entropy corresponds to the optimal partition. The corresponding entropy-like quantities for the other partitions are pseudo-block entropies. The optimal partition is that which maximizes the block entropy (Steuer, 2001).

For this purpose, the threshold $C$ is initially fixed to the mean value of the data in the particular time window. The stationary character of the EM segments under study justifies this choice. For the corresponding symbolic sequence we estimate the associated "pseudo-block entropy". The threshold

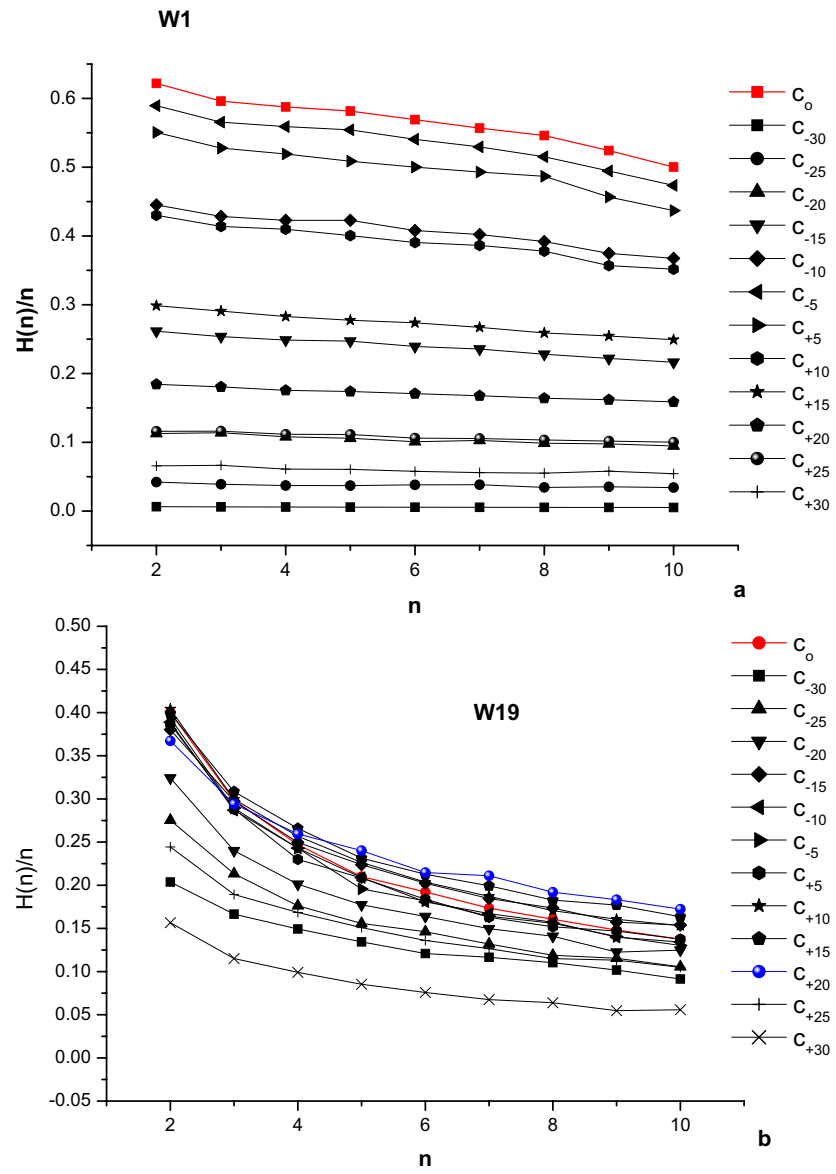

Fig. 4. The choice of the optimal partition. First, the quantity $H(n) / n$ is computed, for various word lengths and for different partitions. The partitioning is performed for different thresholds $C_{i}$, starting from the mean value $C_{o}$. Index $i$, represents the $\%$ deviation from the mean value. The block entropy corresponds to the optimal partition, which is obtained by the maximization of $H(n) / n$. Thus, for the window $\mathrm{W} 1$ (a) the maximization of $H(n) / n$ is done by the partition $C_{o}$ (that is, the mean value). It is concluded that $C_{o}$ corresponds to the optimal partition for the window W1. For the window W19 (b), it seems that the quantity $H(n) / n$ is maximized for $C_{+20}$. However, the differences with the quantity $H(n) / n$ for $C_{o}$ and $C_{+20}$ is very small, in particular in the regime $n \leq 6$ which is interesting for us, see Sect. 4.4.

$C$ is shifted around the mean value. In order to find the real block entropy, which corresponds to the optimal partition, we repeat the above procedure by changing the threshold $C$ around the mean value.

Our analysis indicates that the optimal partition correspond always to a threshold not far from the mean value of the stationary segment. This is depicted in Fig. 4.

Within each stationary time window, the block entropy for the optimal partition serves as a measure of "regularity" of the signal: the lower the value of entropy, the more "ordered" it is. We recall that we follow this procedure in order to show, in a firm mathematical basis, that the complexity of the preseismic signal is significantly reduced as the main catastrophic event is approaching. 


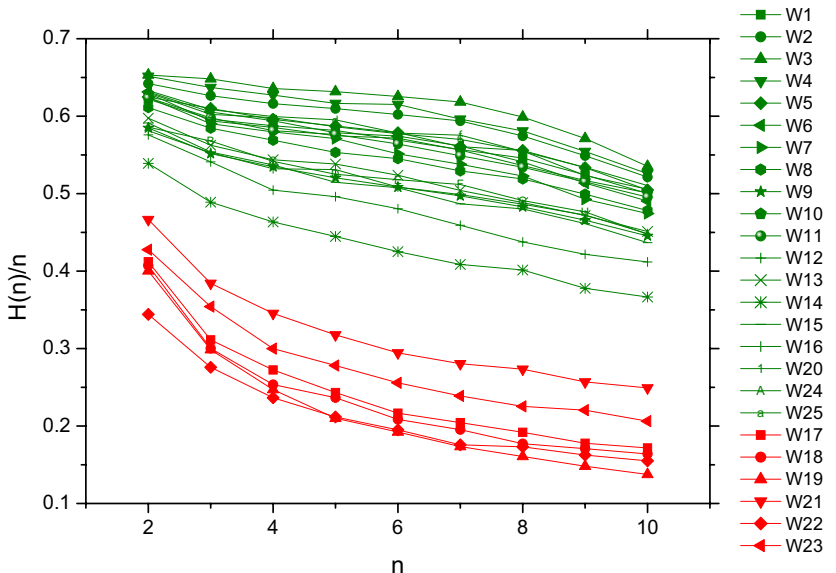

Fig. 5. Block entropy per letter (Eq. 4), as a function of the word length for the stationary windows which have been localized in Fig. 2. We observe a significant reduction of the block entropy per letter during the emergence of the two strong impulsive bursts in the tail of the precursory EM time-series (windows W17-W19 and W21-W23). One can interpret the observed reduction of the block entropy per letter, as a sign of complexity reduction of the signal's underlying fracto-electromagnetic mechanism.

\subsection{The estimated block entropies by lumping}

We then calculate the block entropies by lumping for the optimal partitions of these time windows. In Fig. 5 we depict the block entropy by lumping per letter as a function of the word length for the time windows that we present in Fig. 2. We focus on the time-windows W1-W16 (Fig. 2), namely, from 10 days up to approximately 2 days far from the EQ origin time. The associated group of curves of the block entropy per letter lies in the region of high block entropy values (Fig. 5). The high block entropy values indicate an underlying strong complexity. We note that a complete absence of structure in the signal, would lead to an horizontal line in the block entropy diagram. This is not the present case.

We finally concentrate on the red windows W17, W18, W19 W21, W22, and W23 (Fig. 2). These windows correspond to the two strong impulsive EM bursts in the tail of the precursory emission. We stress that the last impulsive EM emission stopped a few hours before the catastrophic event. The estimated entropies drop to significantly lower values during the emergence of the two strong EM bursts. This behavior witnesses a significant reduction of complexity of the underlying fracto-electromagnetic mechanism: the reduction is of the order of $30 \%$ or more. This evidence may indicate the appearance of a new phase in the tail of the EQ preparation process, which is characterized by a high order of organization.

\subsection{The scaling of block entropies by lumping}

First of all, notice the overall resemblance between Eq. (8) and Einstein's formulation of Boltzmann's formula for the probability of the fluctuation of a macrovariable $A$ in an

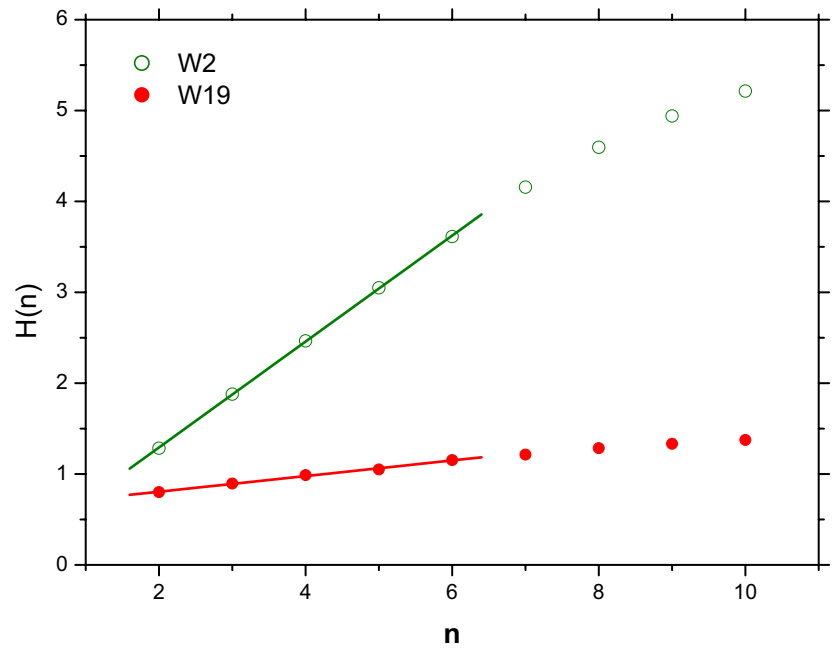

Fig. 6. The observed scaling of the Block Entropy $H(n)$ (Eq. 2), as a function of the word length $n$. The experimental values show a linear best fit for small word lengths with a very good precision. The slope of the line gives the Kolmogorov-Sinai entropy which in $1 D$ coincides with the Lyapunov exponent. This scaling is consistent with the corresponding theoretical predictions, see Nicolis and Gaspard, (1994).

isolated system $P(A) \sim e^{\Delta S(A) / k}$, where $\Delta S$ is the entropy change due to the fluctuation.

McMillan's theorem (see Sect. 3.4) gives us a direct way to speak about the scaling properties of the block entropies. Indeed, the penalization of long words, so important for the preparation of meaningful texts, depends directly on the scaling of the block entropies.

One important conjecture, due essentially to Ebeling and Nicolis (1992) states that the most general (asymptotic) scaling of the block entropies takes the form

$H(n)=e+n h+g n^{\mu_{0}}(\ln n)^{\mu_{1}}$

where $e, h$ and $g$ are constants and $\mu_{0}$ and $\mu_{1}$ are constant exponents.

We attempt to examine the behavior of Eq. (9) for each of the 25 stationary windows under study, depicted in Fig. 2.

In Fig. 6 we present the typical variation of the block entropy by lumping $\mathrm{H}(\mathrm{n})$ as a function of the word length $n$ for two representative windows W2 (green) and W19 (red). If we are restricted to the first six $H(n)$-values, a linear scaling is observed with a great precision. We then perform a least square method for this region and we estimate the slope $h$. Note, that the associated correlation coefficients $(r)$ are close to 1 with a precision better than $10^{-4}$. Working similarly for the rest of the 25 time windows, we conclude that for $n \leq 6$ the same behavior is observed i.e. the equation for the scaling of the block entropy by lumping, is transformed to the simple linear relation

$H(n)=e+n h$

This means that $g=0$ for $n \leq 6$. 


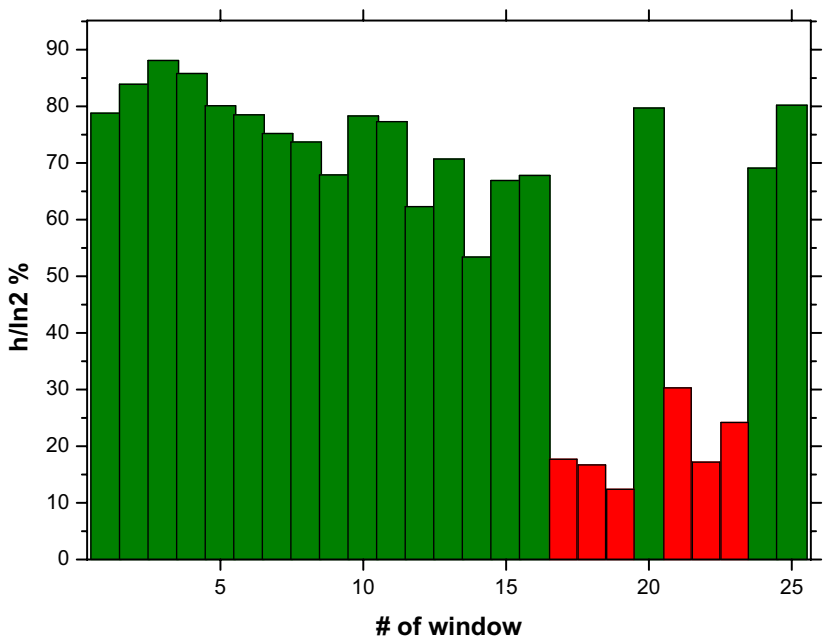

Fig. 7. The normalized Kolmogorov-Sinai entropy (taken as the normalized slope of the linear part of the Block Entropy $H(n)$ ), for the different windows W1-W25. We observe a very important reduction of the entropy inside the two strong impulsive bursts of the precursory EM time series.

We note that when $g=0$ and $h \geq 0$, long words are penalized exponentially (Nicolis and Gaspard, 1994). We focus on the quantity $h$, namely the Kolmogorov-Sinai entropy defined by the slope of Eq. (10). We notice that for a one-dimensional process the Kolmogorov-Sinai entropy coincides with its Lyapunov exponent. The Lyapunov exponent gives a measure of the chaoticity of the signal. For a two letter alphabet, the Kolmogorov-Sinai entropy $h$ takes values from zero to $\ln 2$ (see the discussion in Sect. 7), so that one can normalize dividing by $\ln 2$ and obtain a percentage.

We remark that in Fig. 6 there is a clear-cut distinction of the values of the slopes $h$, that suggest a significant difference in the corresponding processes that generate the sequences W2 (green) and W19 (red) respectively.

In Table 1 we observe that all green blocks have a rather substantial Kolmogorov-Sinai entropy $h$ taking values around $80 \%$. In the opposite, all red blocks take values around $30 \%$ which are significantly lower (see also Fig. 7). This means a high degree of organization for the underlying fracto - electromagnetic emission during the last stage of the precursory EM time-series, possibly related with the nucleation phase of the earthquake (see Sect. 7).

Hence, it is important to note that the linear part of the scaling helps us to the classification of the precursory signals. The question which arises is whether this is an independent algorithmic law of nature. This seems to be an open problem, see the discussion in Sect. 7. However, our results strongly support this hypothesis.

Remark: We restrict ourselves to the region $n \leq 6$, because the maximum statistical accuracy for the block entropies by lumping is of the order of $\ln R$, where $R$ is the total number of points (the length of the window). In our case $R$ is of the order of 4000 , so that $n \leq 8$ and due to the underestimation of
Table 1. The Kolmogorov-Sinai (KS) entropy $h$, the estimated error $\delta h$ and the corresponding percentage $h / \ln 2$ (see Fig. 7) in respect to the maximum value of the KS entropy for the different stationary time windows depicted in Fig. 2.

\begin{tabular}{lccc}
\hline Window No. & $h$ & $\delta h$ & $h / \ln 2(\%)$ \\
\hline W1 & 0.546 & 0.005 & 78.8 \\
W2 & 0.582 & 0.003 & 83.9 \\
W3 & 0.611 & 0.004 & 88.1 \\
W4 & 0.595 & 0.003 & 85.8 \\
W5 & 0.555 & 0.004 & 80.1 \\
W6 & 0.544 & 0.004 & 78.5 \\
W7 & 0.521 & 0.009 & 75.2 \\
W8 & 0.511 & 0.005 & 73.7 \\
W9 & 0.471 & 0.005 & 67.9 \\
W10 & 0.543 & 0.003 & 78.3 \\
W11 & 0.536 & 0.004 & 77.3 \\
W12 & 0.432 & 0.007 & 62.3 \\
W13 & 0.490 & 0.005 & 70.7 \\
W14 & 0.370 & 0.007 & 53.4 \\
W15 & 0.464 & 0.006 & 66.9 \\
W16 & 0.470 & 0.010 & 67.8 \\
W17 & 0.123 & 0.006 & 17.7 \\
W18 & 0.116 & 0.008 & 16.7 \\
W19 & 0.086 & 0.003 & 12.4 \\
W20 & 0.553 & 0.005 & 79.7 \\
W21 & 0.210 & 0.006 & 30.3 \\
W22 & 0.119 & 0.003 & 17.2 \\
W23 & 0.168 & 0.006 & 24.2 \\
W24 & 0.479 & 0.007 & 69.1 \\
W25 & 0.556 & 0.009 & 80.2 \\
\hline
\end{tabular}

the higher entropies we have enough statistical precision for $n \leq 6$.

\subsection{Estimated conditional entropies by lumping}

In Fig. 8 we show the conditional entropies by lumping into some characteristic green and red time windows. In particular for the green windows, which are relatively far from the earthquake origin, the associated conditional entropies are much higher than the corresponding conditional entropies in the red windows, which are near the earthquake occurrence. This behavior may serve as an independent indicator that confirms a significant increase of organization of the underlying fracto-electromagnetic process during the emergence of two strong impulsive signals in the tail of the precursory EM phenomenon. 


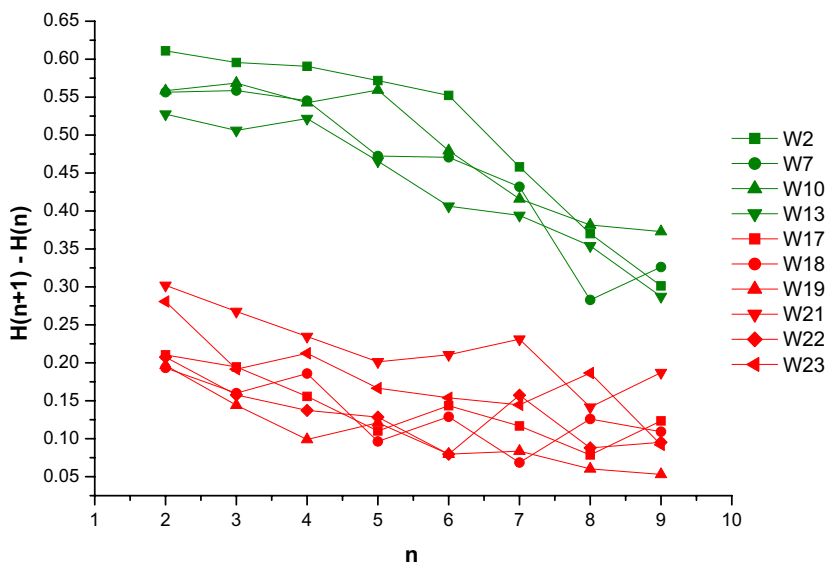

Fig. 8. Conditional entropy $H(n+1)-H(n)$ as a function of the word length for a few typical green and red windows. We observe a significant loss of complexity in the red time windows that correspond to the strong EM bursts in the tail of the precursory emissions (see Fig. 2).

\section{Correlation dimension}

The correlation dimension $D_{2}$ is commonly used to quantify the chaotic structure of time series. The delay-times method is an important tool in non-linear analysis and gives both a qualitative and quantitative measure of the complexity of the time series under examination. It was first established by Grassberger and Procaccia (1983) and is based on the Takens Theorem (Takens, 1981). A time series is constructed from a set of successive and experimentally derived values. From the original time series we then construct a new series, which in this case is composed of delay vectors. For the construction of each of the vectors the estimation of two parameters, the embedding dimension, $m$, and the time-lag, $\tau$, is required. The time-lag represents the window that is used for the computation of the coordinates of these vectors. It is estimated from the decorrelation time, which is the window beyond which the signal ceases to present periodicities. The decorrelation time is calculated either from the first zero-value of the autocorrelation function, or from the first value of the mutual information function (Farmer and Swinney, 1986) that is close to zero. The parameter $\mathrm{m}$ is assigned increasing integer values, in a range that satisfies both the Takens criterion (Takens, 1981) and the maximum admitted window length, according to basic non-linear dynamics theory.

Once the above is completed, the correlation integral (or correlation sum), $C(r)$ is computed for increasing values of $r$. This integral basically computes how many of the delay vectors have a distance between them less than $r$, where $r$ is a ray in the vector space. We are then able to plot $\ln (C)$ vs. $\ln (r)$, where $\ln$ is the natural logarithm function. From this plot we select a scaling region and compute the slope of the curve in that region. This process is repeated for increasing values of the embedding dimension, $\mathrm{m}$, and if the values of the slopes converge, then we have found the cor- relation dimension $D_{2}$ of the time series. The convergence value of the slope is an estimation of the correlation dimension. A time series that results from a complex non-linear dynamic system yields a larger value for the correlation dimension, as opposed to a time series which results from a regular and linear dynamic system, lower correlation dimension values. Generally, the correlation dimension represents the independent degrees of freedom that are required for the proper description of a system or for the construction of its model. When the under estimation time series comes from a stochastic or random process the correlation dimension cannot be estimated as no convergence of the correlation integral slopes can be yielded at all.

\subsection{Correlation dimension estimations}

We calculate the correlation dimension, $D_{2}$, associated with successive segments of 3000 samples each and study the distributions of correlation dimension $D_{2}$ in four consecutive time intervals (Fig. 9). Notice that the third time interval includes the two strong impulsive bursts in the tail of the precursory emission. We concentrate on the fundamental question whether distinguished alterations in associated $D_{2}$ values emerge as Earth's crust failure is approached.

We underline the almost common distributions of the $D_{2}$ values in the first, second and fourth time intervals (Fig. 9). The associated predominance $D_{2}$-values, from 7 up to 10 , indicates a strong complexity. However, we observe a significant decrease of the $D_{2}$-values as we move to the third time window. The observed significant decrease of the $D_{2}$-values signals a strong loss of complexity in the underlying fractoelectromagnetic mechanism during the launching of the two strong EM bursts in the tail of the precursory emission.

We conclude that the analysis in terms of the correlation dimension corroborates the appearance of a new phase in the tail of the EQ preparation process, which is characterized by a higher order of organization.

Remark: In a strict sense, well known problems in estimating $D_{2}$, as well as other measures from short and noisy data segments, would exclude the use of these measure for a characterization of earthquake preparation dynamics. However, one can use the "correlation dimension $\mathrm{D}_{2}$ " in an informal sense as an operational measure of complexity of the preseismic electromagnetic time series (Lehnertz and Elger, 1998). Absolute values of $D_{2}$ are not considered and presumably do not agree with true $D_{2}$ (if it exists). Indeed, only differences with respect to time are assumed reliable and are used to characterize the complex dynamics of the seismogenic electromagnetic activity. In this sense, the values of the correlation dimension estimated here could have the meaning of an "apparent correlation dimension".

We note that, under the above-mentioned restrictions, authors have attempted to evaluate the capability of nonlinear analysis in terms of correlation dimension $D_{2}$ to extract signatures from brain electrical activity predictive of epileptic seizures or from Heat Rate Variability (HRV) data for distinguishing healthy subjects and Coronary Artery Disease 


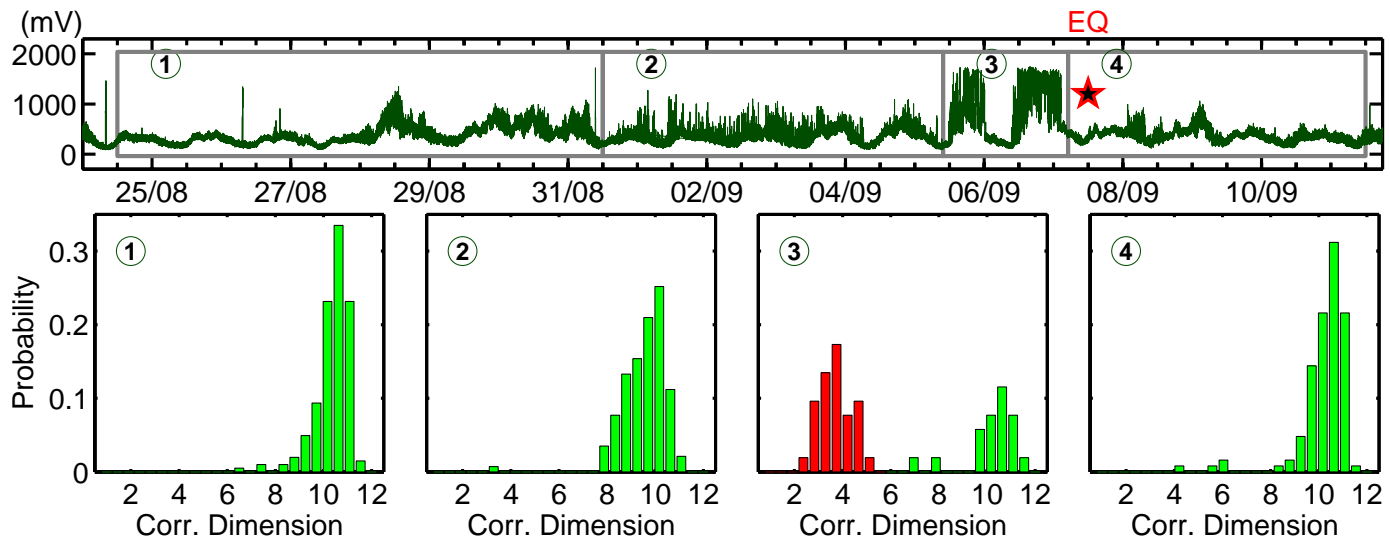

Fig. 9. We first estimate the Correlation Dimension, $D_{2}$, in consecutive segments of 3000 samples each. Then, we trace the distribution of these $D_{2}$-values for four consecutive epochs. The four epochs are depicted in the upper part of the figure. The epochs 1 and 4 correspond to a more or less EM quiescence that precedes and follows respectively the precursory activity. The almost similar distributions in the epochs 1 and 4 characterize the EM background (noise). In epoch 2, the little deformation of the distribution to the left side in respect to the distribution of EM noise indicates that the initial part of the precursory emission is characterized by a little reduction of the complexity, in respect to the high complexity of EM noise. The right lobe that appears in epoch 3 corresponds to the EM background, while the left red lobe seems to correspond to the EM precursory activity. We observe a dramatic shift of the distribution of the $D_{2}$-values in epoch 3 . This evidence indicates a strong reduction of complexity during the emergence of the two strong EM bursts in the tail of the precursory emission.

(CAD). Characteristically, the dynamic system of CAD patients seems to behave more normally represented by a mean correlation dimension $D_{2}=4.2$, while, the healthy subject signals implies a dynamic system with mean correlation $D_{2}=8.4$ (Karamanos et al., 2005). On the other hand, time-resolved analysis of the EEG time series indicates a significant loss of complexity prior to and during seizures (the related effective correlation dimension decrease up to 2), while, far away from any seizure the associated effective correlation dimension varies around 9 (Lehnertz and Elger, 1998). We note the here estimated values of $D_{2}$ are compatible with those associated with HRV and EEG data.

In conclusion, what is really interesting here is not the rigorous mathematics but the changes of the "apparent correlation" $D_{2}$ when we have transitions from one state to another. We applied a time-resolved $D_{2}$ analysis to pre-seismic EM time series recorded during both intervals temporally far away from the catastrophic event under study, as well as in the tail of the earthquake preparation process (nucleation phase): the nucleation phase data files provided less complex statistics.

\section{Precursory signatures in terms of a linear fractal spectral analysis}

The above mentioned results are also supported by a linear fractal spectral analysis. Sufficient experimental and theoretical evidence indicates that as the final failure in the disordered media is approached the underlying complexity manifests itself in linkages between space and time, generally producing patterns on many scales and the emergence of fractal structure close to irreversible phase transitions. Thus, a lot of work on complexity focuses on statistical power laws that describe the scaling properties of fractal processes and structures.

We focus on the statistics of the fluctuations in the preseismic time-series with respect to their amplitude, let's say $A\left(t_{i}\right)$. If the time-series $A\left(t_{i}\right)$ is a temporal fractal that series cannot have a characteristic frequency. The only possibility is then that the power spectrum $S(f)$ has a scaling form:

$S(f) \sim f^{-\beta}$

where $f$ is the frequency of the Fourier Transform (FT). In a $\log S(f)-\log f$ representation the power spectrum is a line with spectral slope $\beta$. The linear correlation coefficient, $r$, is a measure of the goodness of fit to the power law (Eq. 11).

The "global wavelet spectrum" is used in order to provide an unbiased and consistent estimation of the true power spectrum of the time-series. The continuous wavelet transform based on the Morlet wavelet makes the calculation.

The analysis shows that all the EM segments under study (Fig. 2) are fractals, that is, in a $\log S(f)-\log f$ representation the power spectrum is a line with high $r$-value, i.e. from 0.953 to 0.999 . In Fig. 10 we depict the $\log S(f)-\log f$ diagrams for two green and two red time windows.

The fact that the data follow the power-law (Eq. 11) suggests that the pre-seismic EM emission could be ascribed to a multi time-scale cooperative activity of numerous activated cracks in which an individual unit's activity is dominated by its neighbors so that all units simultaneously alter their behavior to a common fractal pattern. In order to examine transient phenomena, we study how the exponent $\beta$ evolve as the global failure is approached. The result is that into green time windows $\beta$ lies from 1.25 to 1.85 , while, into red windows we have that $\beta$ is between 2.56 to 2.92 .

The estimated $\beta$-values $(1<\beta<3)$ corroborate to the presence of memory in the underlying fracto-EM process. The 

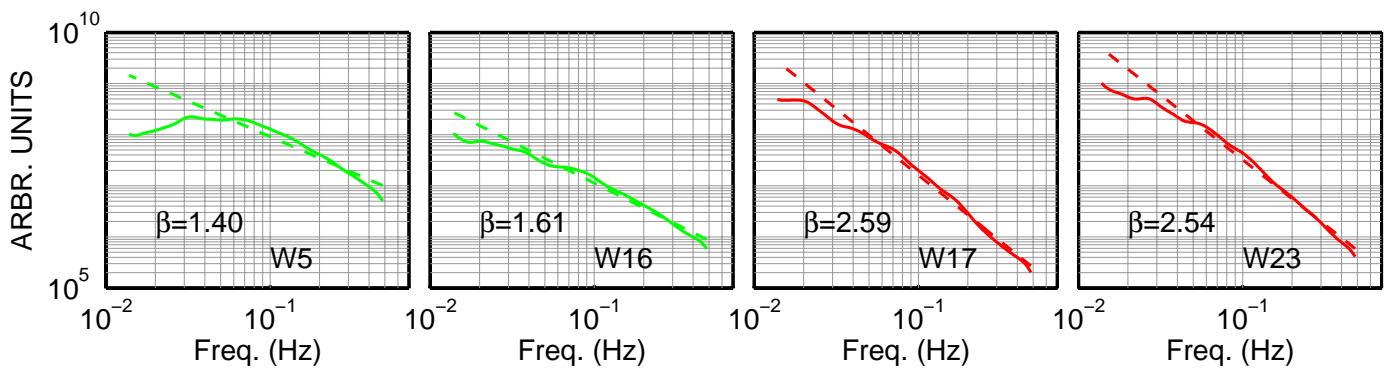

Fig. 10. A $\log$ - $\log$ representation of the relation $S(f)=f^{-\beta}$ for two typical green and two red windows. We observe that this equation fits very well. The associated $\beta$-values inform that the green windows follow anti-persistent behavior, while the red ones are characterized by persistent properties.

EM fluctuations show strong correlations with previous ones, i.e. the system refers to its history in order to define its future (non-Markovian behavior).

We note that Schwarz et al. (1998) have applied a relevant method in a very similar way to classify signals of solar wave bursts.

\subsection{The transition from the anti-persistent to the persistent} regime

Two classes of signal have been widely used to model stochastic fractal time series (Henegham and McDarby, 2000): fractional Gaussian noise (fGn) and fractional Brownian motion (fBm). These are, respectively, generalizations of white Gaussian noise and Brownian motion. A formal mathematical definition of continuous $\mathrm{fBm}$ was first offered by Mandelbrot and Ness (1968). For the case of the fBm model the scaling exponent $\beta$ lies between 1 and 3 , while the regime of fGn is indicated by $\beta$-values from -1 to 1 (Henegham and McDarby, 2000). The $\beta$-values in the VHF EM pre-fracture time series are distributed in the region from 1 to 3 . This means that the seismogenic EM activity follows the $\mathrm{fBm}$ model.

The $\beta$-exponent is related to the Hurst exponent, $H$, by the formula

$\beta=2 H+1 \quad$ with $\quad 0<H<1$

for the fBm model (Mandelbrot and Ness, 1968; Henegham and McDarby, 2000).

The exponent $H$ characterizes the persistent/antipersistent properties of the signal (Eftaxias et al., 2004, and references therein). The range $0<H<0.5,(1<\beta<2)$ indicates an anti-persistency, reflecting that increases in the value of a time-series are likely to be followed by decreases and conversely. Physically, this implies a set of fluctuations tending to induce a stability to the system, namely a nonlinear feedback system that "kicks" the opening rate away from extremes. The group of the 19 green time windows show anti-persistent behavior (the Hurst exponent lies from 1.25 to 1.85 ). On the contrary, the time-series has persistent properties (the Hurst exponent range from 2.56 to 2.92) during the red time windows, namely, within the two strong EM bursts. This means that increases in the value of a time-series are likely to be followed by increase, namely the system has been starting to self-organize by a positive feedback process. The system seem to acquire to a great degree the property of irreversibility. Thus the launch of the persistent activity could give a significant hint of a considerable probability for a forthcoming significant seismic event. The appearance of persistent properties within the characteristic two EM bursts in the tail of the VLF EM precursor, i.e. a few hours before the EQ occurrence, strongly supports the concept that the emergence of the two impulsive bursts witnesses the appearance of the final preparation phase of the impending $\mathrm{EQ}$.

It is worth mentioning that laboratory experiments by means of acoustic and EM emission also show that the main rupture occurs after the appearance of persistent behavior (Ponomarev et al., 1997; Alexeev and Egorov, 1993; Alexeev et al., 1993; Lei et al., 2004). Recently, Sammis and Sornette (2002) presented the most important mechanisms for such positive feedback mechanism.

\section{Discussion and conclusions}

In this paper, we are studying in terms of nonlinear and linear techniques, whether precursory EM features emerged indicating the approach to the global failure. More precisely, we presented an analysis of pre-seismic EM emission associated with the Athens EQ. In order to extract possible precursory signatures we analyze the recorded preseismic EM time series into distinct time windows. The aim was to discover a clear difference of dynamical characteristics as the catastrophic event was approaching.

Our study was firstly based on symbolic dynamics and Shannon-like entropy. For this purpose, starting from the raw data, we isolated stationary windows in the detected preseismic EM time series. Within each of these stationary time windows the block entropy by lumping for the optimal partition has been calculated. The block entropy was interpreted as a measure of "complexity". Dynamical changes with time in the precursory EM activity were clearly visible. In particular, the analysis helps to discriminate two characteristic preseismic epochs: the second epoch abruptly emerged a few 
tens of hours before the earthquake occurrence and abruptly ended a few hours before the global instability. This epoch is of much lower complexity than the early first epoch. The existence of two distinct periods during the evolution of the preseismic EM activity, and particularly the significant reduction of the complexity into the second epoch is also verified by the analysis in terms of Kolmogorov-Sinai entropy, as well as by the calculation of the conditional entropies. The appearance of a new phase, which is characterized by a strong loss of complexity, in the tail of the precursory EM activity, is also verified in terms of correlation dimension $D_{2}$.

Finally, we turn into the linear method. In order to extract transient precursory footprints, we divide the measurements into short consecutive time-windows and analyze these in terms of statistical fractal analysis. The dynamics into each time-window is mainly characterized by the value of the Hurst exponent $H$, which characterizes the persistent/antipersistent properties of the signal. Based on the behavior of Hurst exponents we independently can discriminate two characteristic epochs in the evolution of precursory activity: The first epoch includes the initial anti-persistent part of the precursory time series, i.e. the epoch with $0<H<0.5$. This behavior implies a set of fluctuations tending to induce stability within the system, namely, a nonlinear negative feedback, which kicks the system away from extremes. In the opposite, the second epoch includes the last stage of the precursory activity characterized by persistent behavior, i.e. epoch with $0.5<H<1$. This implies that a new phase has been emerged, which is characterized by a super-diffusion behavior (or a nonlinear positive feedback mechanism).

Theoretical studies suggest that final EQ and neuralseizure dynamics should have many similar features and could be analyzed within similar mathematical frameworks (Kapiris et al., 2005 and references therein). Recently (Kapiris et al., 2005), by monitoring the temporal evolution of fractal spectral characteristics in EEG (electroencephalograph) recordings on rat experiments and pre-seismic electromagnetic (EM) time series associated with the Athens EQ, we showed that many similar distinctive symptoms (including common alterations in their associated scaling parameters) emerge as epileptic seizures and EQs are approaching. We emphasize that both catastrophic events happen after the occurrence of persistent behavior: the transition from antipersistent to persistent behavior may indicate that the onset of a severe crisis is imminent.

We stress that the first epoch of high complexity corresponds to anti-persistent behavior and the second epoch of low complexity corresponds to persistent behavior. We think that, taken together: the significant reduction of the "blockentropy", the reduction of the Kolmogorov-Sinai entropy, the considerable decrease of "correlation dimension", and the emergence of strong persistent behavior, it might be concluded that they are generated by the last stage, i.e. nucleation phase, of the impending EQ. Sufficient field and laboratory experimental evidence indeed supports the above consideration.
1. The first strong EM burst contains approximately $20 \%$ of the total EM energy received during the emergence of the two bursts, and the second the remaining 80\% (Eftaxias et al., 2001). On the other hand, the fault modelling of the Athens EQ, based on information obtained by radar interferometry (Kontoes et al., 2000), predicts two faults: the main fault segment is responsible for $80 \%$ of the total energy released, with the secondary fault segment for the remaining $20 \%$. A recent seismic data analysis carried out by M. Kikuchi, using the now standard methodology (Kikuchi and Kanamori, 1990), indicates that a two-event solution for the Athens EQ is more likely than a single event solution (Eftaxias et al., 2001). According to Kikuchi, there was probably a subsequent $\left(M_{w}=5.5\right) \mathrm{EQ}$ after about $3.5 \mathrm{~s}$ of the main event $\left(M_{w}=5.8\right)$. This surprising correlation in the energy domain between the two strong pre-seismic $\mathrm{kHz}$ EM signals and two faults activated in the case of the Athens EQ, strongly supports the hypothesis that the two strong EM bursts reveal the nucleation of the impending EQ. On 1 and 2 September 1999, a series of ULF seismic electric signals (VAN-signals) has been recorded with duration of $9 \mathrm{~h}$ (Varotsos et al., 1999). The recorded activity exhibits the following peculiarity: its last portion has a larger amplitude and also changes polarity (see Fig. 3 in Varotsos et al., 1999). The detected precursory anomaly could be interpreted as consisting of two separate activities, coming from two different sources (Varotsos et al., 1999). It might be argued that the information for the impending two faults activation had imprint in the detected VAN-signals as well.

2. Enhanced thermal infrared (TIR) emission from the Earth's surface are being retrieved by satellites prior to earthquakes, also known as "thermal anomalies", has been frequently reported (Tronin et al., 2002; Ouzounov and Freund, 2004). Recently, (Freud et al., 2005) have proposed an explanation for "thermal anomalies" from a solid state physics viewpoint, namely that the IR emission giving rise to the apparent land surface temperature fluctuations is due to the radiative decay of vibrationally highly excited $O-O$ bonds, which form at the rock surface during recombination of positive holes, activated by the build-up of stress in the Earth's crust. Clear increase in the TIR emission over the area around the Athens' EQ epicenter has been detected from satellites during the days where the EM emission of low complexity and persistent behavior emerged (Filizzola et al., 2004).

3. Recently, we have studied the preseismic EM signals in terms of the following model (Contoyiannis et al., 2005): the focal area consists of (a) a backbone of strong and almost homogeneous large asperities that sustains the system and (b) a strongly heterogeneous medium that surrounds the family of strong asperities. We suggest that the anti-persistent part of the precursory EM activity finds his origin in the fracture of the 
heterogeneous component, while, the persistent part is caused by the fracture of asperities that sustain the system.

4. Laboratory studies under well-controlled conditions i.e. using well-prepared samples containing well-known asperities should be useful for understanding the physics of asperities. Recently (Lei et al. 2000, 2004) have studied how an individual asperity fractures, how coupled asperities fracture, and also the role of asperities in fault nucleation and as potential precursors prior to dynamic rupture. These observations reveal a strong similarity between the fracture of asperities in laboratoryscale experiments and tectonic-scale events. More precisely, they suggest the following.

(a) Intense micro-cracking may occur in a strong asperity when the local stress exceeds the fracture stress of the asperity. This feature is in agreement with our results.

(b) The self-excitation strength, which expresses the influence of excitation of an event on succeeding events or, equivalently, the degree of positive feedback in the dynamics, reaches a maximum of $\sim 1$ during the nucleation phase of the fault. Recall that the $H$ exponent also approaches its maximum value of 1 in the tail of the precursory EM radiation.

(c) The fractal dimension decreases from $\sim 2.2$ in the pre-nucleation phase to $1.0-1.4$ during asperity fracture. The authors correlate the decrease of the fractal dimension with the concentration of stresses around the family of asperities. The fractal dimension $d$ of the detected, in the field, EM time series is found from the relation $d=2-H$ for the fBm class. After considering the observed values of the exponents $\beta$ (or $\mathrm{H}$ ) (see Sect. 6) we conclude that the time series exhibits a fractal dimension from $\sim 1.2$ to $\sim 1$, in its tail, i.e. within the precursory two strong EM bursts. On the other hand a few days prior to the Athens event, the seismicity was centered at the epicenter area, i.e. at a distance of about one source dimension from the Athens EQ epicenter (Tzanis and Makropoulos, 2002; Papadopoulos, 2002).

Although significant problems remain when extrapolating laboratory results to field conditions, the above mentioned experimental findings might indicate that the emergence of strong positive correlations, reflect the faulting nucleation phase of the EQ preparation, namely, the fracture of the sequence of asperities

5. The statistical analysis reveals that the cumulative number $(N>A)$ of EM events detected prior to the Athens EQ-namely, the number of EM events having amplitude larger than $A$, follows the power law $N(>A) \sim A^{-0.62}$ (Kapiris et al., 2004a) . Rabinovitch et al. (2002) have studied the fractal nature of EM radiation induced in rock fracture. The analysis of the pre-fracture EM time series reveals that the cumulative distribution of the amplitudes also follows a power law with exponent 0.62 . The accord of the critical exponents suggests that the same fracto-electrodynamics may hold from the geophysical down to the microscopic scale. In addition, scaling similarities of acoustic and EM emissions during multiple fractures in solid materials from the laboratory scale up to the geophysical scale strongly support the hypothesis that both types of emission are generated during crack opening (Kapiris et al., 2004a).

The question to what extend the present study may contribute to the prediction of EQs, is not easily answered. The wide diversity of different types of EQ preparation process makes it even unlikely that there exists one solution at all. In this spirit, we wonder if the observed significant reduction of the block entropies by lumping (around 30\%) just before the EQ, followed by the emergence of persistent properties, is or not a new statistical law of nature. In our opinion more experimentation could enlighten this point. We hope that the present work might contribute to further research about a more fundamental understanding of EQs generation mechanism. As concern to basic research, it remains to be established whether different methods of nonlinear and time series analysis could furnish additional precursors that allow one to extend the knowledge about earthquake generating mechanisms.

Acknowledgements. The authors would like to thank very much the anonymous referees for their constructive criticism and useful remarks. The authors also acknowledge G. Nicolis, J. S. Nicolis and I. Kotsireas for fruitful discussions. For the entropy computations, the authors used the program ENTROPA by V. Basios (Universite Libre de Bruxelles). All the entropy computations of this paper have been performed remotely at the CoLab, Center for Experimental and Constructive Mathematics (CECM), Simon Fraser University, Barnaby, Canada and we are grateful to J. M. Borwein for giving access to this account.We gratefully acknowledge financial support by the European Union under contract EPEAEK/PYTHAGORAS 70/3/7357.

Edited by: J. Kurths

Reviewed by: two referees

\section{References}

Alexeev, D. and Egorov, P.: Persistent cracks accumulation under loading of rocks and ccncentration criterion of failure, Reports of RAS, 333, 6, 769-770, in Russian, 1993.

Alexeev, D., Egorov, P., and Ivanov, V.: Hurst statistics of time dependence of electromagnetic emission under rocks loading, Physical-Technical problems of exploitation of treasures of the soil, 5, 27-30, in Russian, 1993.

Bahat, D., Frid, V., Rabinovitch, A., and Palchik, V.: Exploration via electromagnetic radiation and fractographic methods of fracture properties induced by compression in glass-ceramic, Int. J. Fract., 116, 179-194, 2002. 
Contoyiannis, Y., Kapiris, P., and Eftaxias, K.: Monitoring of a preseismic phase from its electromagnetic precursors, Phys. Rev. E, 71, 066123, 2005.

Dickinson, J., Langford, S., Jensen, L., McVay, G., Kelso, J., and Pantano, C.: Fractoemission from fused silica and sodium silicate glasses, J. Vac. Sci. Technol. A, 6, 1084-1089, 1988.

Ebeling, W. and Nicolis, G.: Word frequency and entropy of symbolic sequences: A dynamical Perspective, Chaos, Solitons \& Fractals, 2, 635-650, 1992.

Eftaxias, K., Kapiris, P., Polygiannakis, J., Bogris, N., Kopanas, J., Antonopoulos, G., Peratzakis, A., and Hadjicontis, V.: Signatures of pending earthquake from electromagnetic anomalies, Geophys. Res. Lett., 28, 3321-3324, 2001.

Eftaxias, K., Kapiris, P., Dologlou, E., Kopanas, J., Bogris, N., Antonopoulos, G., Peratzakis, A., and Hadjicontis, V.: EM anomalies before the Kozani earthquake: A stady of their behavior through laboratory experiments, Geophys. Res. Lett., 29, 69/1-69/4, 2002.

Eftaxias, K., Frangos, P., Kapiris, P., Polygiannakis, J., Kopanas, J., Peratzakis, A., Skountzos, P., and Jaggard, D.: Review and a Model of Pre-Seismic electromagnetic emissions in terms of fractal electrodynamics, Fractals, 12, 243-273, 2004.

Farmer A. and Swinney, H.: independent coordinates for strange attractors from mutual information, Phys. Rev. A, 33, 1134-1140, 1986.

Filizzola, C., Pergola, N., Pietrapertosa, C., and Tramutoli, V.: Robust satellite techniques for seismically active areas monitoring: a sensitivity analysis on 7 September 1999 Athens earthquake, Phys. Chem. Earth, 29, 517-527, 2004.

Freund, F. T., Keefner, J., Mellon, J. J., Lau, La, A., and Ouzounov, D.: Enhanced mid-infrared emission from igneous rocks under stress, Geophys. Res. Abstr., 7, 09568, 2005.

Frid, V., Rabinovitch, A., and Bahat, D.: Electromagnetic radiation associated with induced triaxial fracture in granite, Phil. Mag. Lett., 79, 79-86, 1999.

Frid, V., Bahat, D., Goldbaum, J., and Rabinovitch, A.: Experimental and theoretical invesrigations of electromagnetic radiation induced by rock fracture, Isr. J. Earth Sci., 49, 9-19, 2000.

Fukuda, K., Nunes, L., and Stanley, H.: Similarities between communication dynamics in the Internet and the automatic nervous system, Europhys. Lett., 62, 189-195, 2003.

Grassberger, P. and Procaccia, I.: Characterization of strange attractors, Phys. Rev. Lett., 50, 346-349, 1983.

Gershenzon, N. and Bambakidis, G.: Modelling of seismoelectromagnetic phenomena, Russ. J. Earth Sci., 3, 247-275, 2001.

Gonzales, A. and Pantano, C.: Fractoemission during crack propagation in glass, Appl. Phys. Lett., 57, 246-248, 1990.

Hayakawa, M.: Atmospheric and Ionospheric Electromagnetic Phenomena Associated with Earthquakes, Terrapub, Tokyo, 1999.

Hayakawa, M. and Molchanov, O.: Seismo Electromagnetics, Terrapub, Tokyo, 2002.

Henegham, C. and McDarby, G.: Establishing the relation between detrended fluctuation analysis and power spectral density analysis foe stochastic processes, Phys. Rev. E, 62, 6103-6110, 2000.

Kapiris, P., Balasis, G., Kopanas, J., Antonopoulos, G., Peratzakis, A., and Eftaxias, K.: Scaling similarities of multiple fracturing of solid materials, Nonlin. Processes Geophys., 11, 137-151, 2004a, SRef-ID: 1607-7946/npg/2004-11-137.

Kapiris, P., Eftaxias, K., and Chelidze, T.: The electromagnetic signature of prefracture criticality in heterogeneous media, Phys. Rev. Lett., 92, 065702/1-4, 2004b.
Kapiris, P., Polygiannakis, J., Li, X., Yao, X., and Eftaxias, K.: Similarities in precursory features in seismic shocks and epileptic seizures, Europhys. Lett., 69, 657-663, 2005a.

Kapiris, P., Nomicos K., Antonopoulos G., Polygiannakis J., Karamanos K., Kopanas J., Zissos A., Peratzakis A., and Eftaxias K.: Distinguished seismological and electromagnetic features of the impending global failure: did the 7 September 1999 M5.9 Athens earthquake come with a warning?, Earth Planets and Space, 57, 215-230, 2005b

Karamanos, K. and Nicolis, G.: Symbolic Dynamics and Entropy Analysis of Feigenbaum Limit Sets, Chaos, Solitons \& Fractals, 10, 1135-1150, 1999

Karamanos, K.: Entropy analysis of substitutive sequences revisited, J. Phys. A: Math. Gen., 34, 9231-9241, 2001.

Khinchin A. I.: Mathematical Foundations of Information Theory, Dover, New York, 1957.

Kikuchi, M. and Kanamori, H.: Inversion of complex body waves III, Bull. Seism. Soc. Am., 81, 2335-2350, 1990.

Kontoes, C., Elias, P., Sycioti, O., Briole, P., Remy, D., Sachpazi, M., Veis, G., and Kotsis, I.: Displacement field and fault model for the September 7, Athens earthquake inferred from the ERS2 satellite radar interferometry, Geophys. Res. Lett., 27, 39893992, 2000.

Langford, S., Doering, D., and Dickinson, J.: Production of free charge carriers during fracture of single-crystal silicon, Phys. Rev. Lett., 59, 2795-2798, 1987.

Lehnertz, K., Elger C.: Can epileptic seizures be predicted?, Evidence from nonlinear time series analysis of brain electrical activity, Phys. Rev. Lett., 80, 5019-5022, 1998.

Lei, X., Nishizawa, O., Kusunose, K., Cho, A., Satoh, T., and Nishizawa, O.: Compressive failure of mudstone samples containing quartz veins using rapid ae monitoring: the role of asperities, Tectonophysics, 328, 329-340, 2000.

Lei, X., Masuda, K., Nishizawa, O., Jouniaux, L., Liu, L., Ma, W., Satoh, T., and Kusunose, K.: Detailed analysis of acoustic emission activity during catastrophic fracture of faults in rock, J. Struct. Geol., 26, 247-258, 2004.

Li, H., Jia, Z., Bai, Y., Xia, M., and Ke, F.: Damage localization, sensitivity of energy release and the catastrophe transition, Pure Appl. Geophys., 159, 1933-1950, 2002.

Mandelbrot, B. and Ness, J.: Fractional Brownian motions, fractional noises and applications, SIAM Rev., 10, 422-437, 1968.

Mavromatou, C., Hadjicontis, V., Ninos, D., Mastroyiannis, D., Hadjicontis, E., and Eftaxias, K.: Understanding the fracture phenomena in inhomogeneous rock samples and ionic crystals, by monitoring the electromagnetic emission during the deformation, Phys. Chem. Earth, 29, 353-357, 2004.

Miura, T. and Nakayama, K.: Spectral analysis of photons emitted during scratching of an insulator surface by a diamond in air, J. Appl. Phys., 88, 5444-5447, 2000.

Morgounov, V.: Relaxation creep model of impending earthquake, Annali di Geofisica, 44, 369-381, 2001.

Nicolis, G.: Introduction to Nonlinear Science, Cambridge University Press, Cambridge, 1995.

Nicolis, G. and Gaspard, P.: Toward a probabilistic approach to complex systems, Chaos, Solitons \& Fractals, 4, 41-57, 1994.

Nicolis, G., Rao, G., Rao J., and Nicolis, C.: Generation of spatially asymmetric, information-rich structures in far from equilibrium systems, in: Coherence and Chaos in Dynamical Systems, edited by: Christiansen, P. L. and Parmentier, R. D., Manchester University Press, 1988. 
Nicolis, G., Nicolis, C., and Nicolis, J. S.: Chaotic Dynamics, Markov partitions and Zipf's law, J. Stat. Phys., 54, 915-924, 1989.

Nicolis, J. S.: Chaos and Information Processing, World Scientific, Singapore 1991.

Nikolopoulos, S., Kapiris, P., Karamanos K., and Eftaxias, K.: A unified approach of catastrophic events, Nat. Hazards Earth Syst. Sci., 4, 615-637, 2004, SRef-ID: 1684-9981/nhess/2004-4-615.

Ouzounov, D. and Freund, F. T.: Mid-Infrared emission prior to strong earthquakes analyzed by remote sensing data, Adv. Space Res, 33, 268-273, 2004.

Papadopoulos, G.: The Athens, Greece earthquake (Ms 5.9) of 7 September 1999: an event triggered by the Izmit, Turkey, 17 August 1999 earthquake?, Bull. Seism. Soc. Am., 92, 312-321, 2002.

Ponomarev, A., Zavyalov, A., Smirnov, V., and Lockner, D.: Physical modelling of the formation and evolution of seismically active fault zones, Tectonophysics, 277, 57-81, 1997.

Rabinovitch, A., Bahat, D., and Frid, D.: Similarity and Dissimilarity of electromagnetic radiation from carbonate rocks under compression, drilling and blasting, International Journal of Rock Mechanics \& Mining Sciences, 39, 125-129, 2002.

Sammis, C. and Sornette, D.: Positive feedback, memory, and the predictability of earthquakes, PNAS, 99, 2501-2508, 2002.

Schwarz, U., Benz, A.O., Kurths, J., and Witt A.: Analysis of solar spike events by means of symbolic dynamics methods, Astron. Astrophys., 277, 215-224, 1993.

Schwarz, U., Kurths, J., Kliem, B., Kruger, A., and Urpo, S.: Multiresolution analysis of solar mm-wave bursts, Astron. Astrophys. Suppl. Ser., 127, 309-318, 1998.
Sharon, E. and Fineberg, J.: Microbranching instability and the dynamic fracture of brittle materials, Phys. Rev. B, 54, 7128-7138, 1996.

Sharon, E., Gross, S., and Fineberg, J. : Local crack branching as a mechanism for instability in dynamic fracture, Phys. Rev. Lett., 74, 5096-5099, 1995.

Sharon, E. and Fineberg, J.: Confirming the continuum theory of dynamic brittle fracture for fast cracks, Nature, 397, 333-335, 1999.

Sornette, D.: Critical Phenomena in Natural Sciences, Chaos, Fractals, Self-organization and Disorder: Conceptsand Tools, Second edition, Springer Series in Synergetics, Heidelberg, 2004.

Steur, R., Molgedey, L., Ebeling, W., and Jimenez-Montano, M. A.: Entropy and optimal partition for data analysis, Eur. Phys. J. B, 19, 265-269, 2001.

Takens, F.: Detecting strange attractors in turbulence, in Dynamical systems and turbulence, Warwick 1980: Proceedings of symposium held at the university of Warwick 1979-1980, SpringerVerlag, Berlin, 366-381, 1981.

Tronin, A., Hayakawa, M., and Molchanov, O.: Thermal IR satellite data application for earthquake research in Japan and China, J. Geodyn., 33, 519-534, 2002.

Tzanis, A. and Makropoulos, K.: Did the 7 September 1999 M5.9 Athens earthquake come with a warning?, Natural Hazards, 27, 85-103, 2002.

Varotsos, P., Eftaxias, K., Hadjicontis, V., Bogris, N., Skordas, E., Kapiris, P., and Lazaridou, M.: Three notes on the extent of the SES sensitive area around Lamia (LAM), Greece, Acta Geophys. Polonika, XLVII, 435-443, 1999. 